Supporting Information for the Manuscript

\title{
Coverage-Dependent Adsorption Geometry of Acetophenone on Pt(111)
}

Smadar Attia and Swetlana Schauermann*

Institute of Physical Chemistry, Christian-Albrechts-University Kiel,

Max-Eyth-Str. 2, 24118, Kiel, Germany

Corresponding author: schauermann@pctc.uni-kiel.de

\section{Temperature dependence of acetophenone at multilayer coverages.}

The effect of annealing a surface pre-covered by multilayers of acetophenone at $115 \mathrm{~K}$ to various temperatures is displayed in Fig. S1. These experiments were performed by heating to the indicated temperature for a period of $2 \mathrm{~s}$ and allowing the sample to cool once again to $115 \mathrm{~K}$, following which the infrared spectrum was recorded.

Little change is observed on slowly warming the surface to $135 \mathrm{~K}$, but a distinct transition occurred in the 135-153 K region, generating a spectrum with narrower bands as well as different intensity distribution. The broad, rounded peaks of the spectrum recorded at $115 \mathrm{~K}$ is typical of those for amorphous multilayers, whereas the sharper narrower features of the two upper most spectra (e.g 153 and $170 \mathrm{~K}$ ) suggest a crystalline multilayer. The results might point to crystallization of the initially amorphous ice. Determination of acetophenone molecule at submonolayer coverages presented in this study is thus based on comparison of IR spectra of multilayers of acetophenone obtained at $115 \mathrm{~K}$.

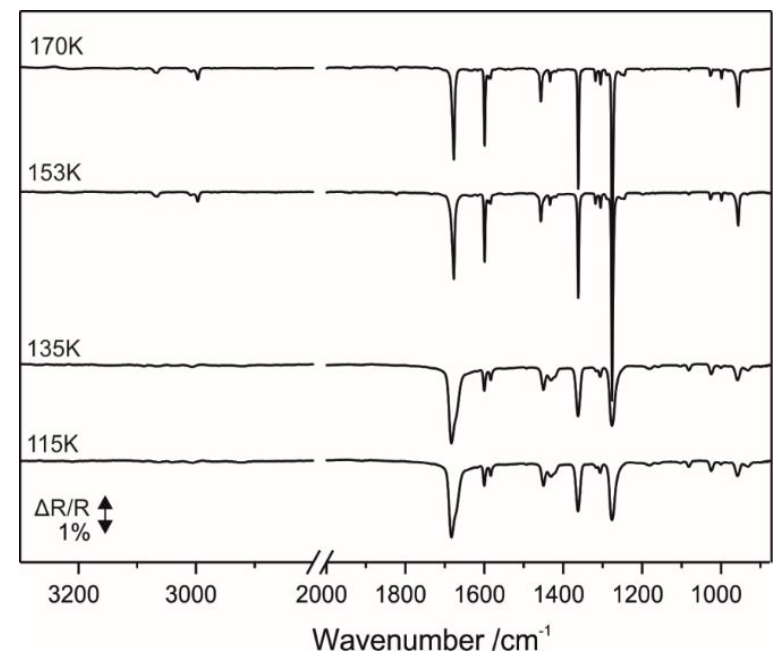

Figure S1: IRAS spectra of multilayers of acetophenone adsorbed on clean $\operatorname{Pt}(111)$ as a function of annealing temperature. The annealing temperatures are marked adjacent to the corresponding spectra. All spectra were recorded at $115 \mathrm{~K}$. 


\section{Initial Stages of Acetophenone Adsorption at $115 \mathrm{~K}$}

Figure S2 shows the evolution of vibrational spectra after acetophenone exposure at $115 \mathrm{~K}$. The spectra are the 5 uppermost spectra from the Figure 3 plotted in the vibrational range $1950-900$ $\mathrm{cm}^{-1}$. It can be clearly seen that the evolution of the vibrational bands and their intensity distributions is very similar to that one observed for the deposition temperature $155 \mathrm{~K}$ shown in Figure 4 in the manuscript. This observation suggests that the same surface species are formed at both investigated temperatures in the low coverage conditions, which are indicated in the manuscript as the species S1.

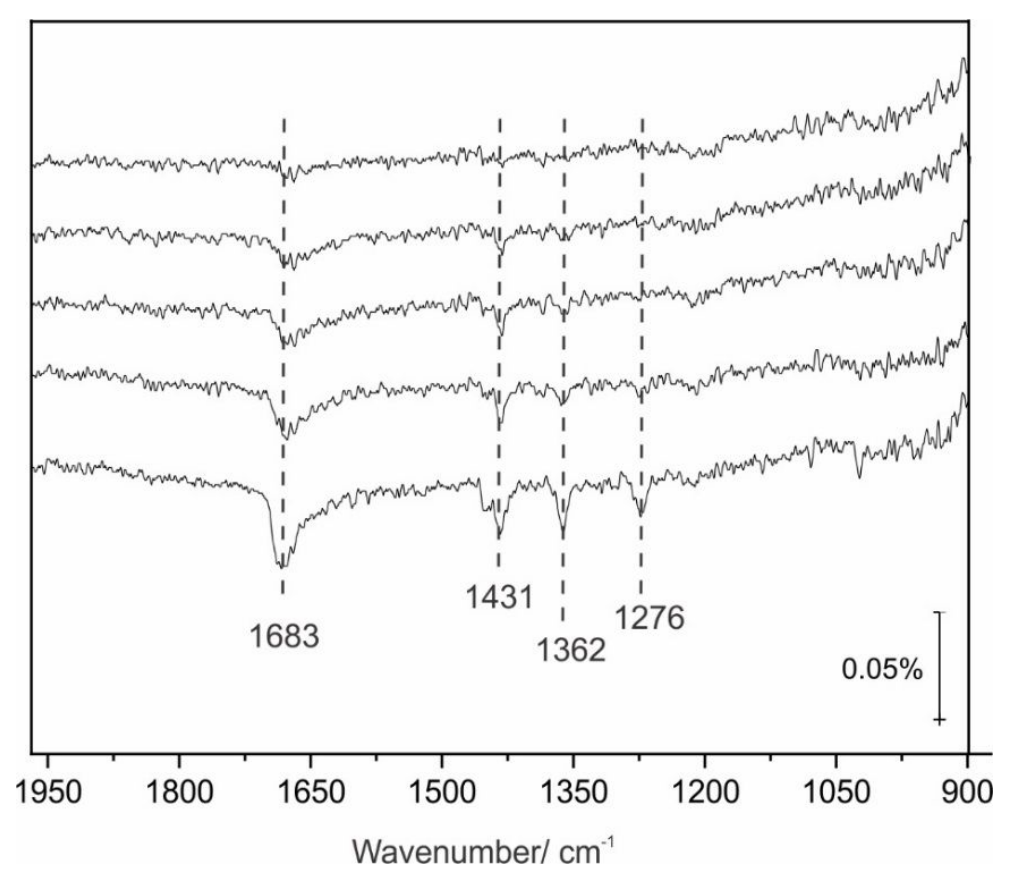

Figure S2: IR spectra of acetophenone on $\mathrm{Pt}(111)$ recorded at $115 \mathrm{~K}$ as a function of exposure time of acetophenone beam $\left(3 \times 10^{12} \mathrm{~cm}^{-2} \mathrm{~s}^{-1}, \Delta \mathrm{t} 110 \mathrm{sec}\right)$. The figure represents the uppermost spectra shown in Fig. 3 of the manuscript. 